\title{
First Order Mem-Circuits: Modeling, Nonlinear Oscillations and Bifurcations
}

\author{
Ricardo Riaza
}

\begin{abstract}
This paper presents a theoretical framework intended to accommodate circuit devices described by characteristics involving more than two fundamental variables. This framework is motivated by the recent appearance of a variety of so-called mem-devices in circuit theory, and makes it possible to model the coexistence of memory effects of different nature in a single device. With a compact formalism, this setting accounts for classical devices and also for circuit elements which do not admit a two-variable description. Fully nonlinear characteristics are allowed for all devices, driving the analysis beyond the framework of Chua and Di Ventra et al. We classify these fully nonlinear circuit elements in terms of the variables involved in their constitutive relations and the notions of the differential- and the state-order of a device. We extend the notion of a topologically degenerate configuration to this broader context, and characterize the differential-algebraic index of nodal models of such circuits. Additionally, we explore certain dynamical features of memcircuits involving manifolds of non-isolated equilibria. Related bifurcation phenomena are explored for a family of nonlinear oscillators based on mem-devices.
\end{abstract}

Index Terms - nonlinear circuit, memristor, memcapacitor, meminductor, nodal analysis, differential-algebraic equation, index, nonlinear oscillator, bifurcation.

EDICS: NOLIN100, ACS100.

\section{INTRODUCTION}

Broadly speaking, nonlinear circuit theory is concerned with the study of constrained ordinary differential equations involving time and four $m$-dimensional variables $q$, $\varphi, i, v$ (standing for charge, flux, current and voltage, respectively), with the following restrictions:

(a) the $2 m$ differential relations $q^{\prime}=i, \varphi^{\prime}=v$ always hold;

(b) the vectors $v, i$, satisfy a total amount of $m$ linearly independent relations $B v=0, D i=0$ coming from Kirchhoff laws;

(c) the characteristics of devices define $m$ additional relations among the circuit variables.

This means that nonlinear circuit models can be generally written in the form

$$
\begin{aligned}
q^{\prime} & =i \\
\varphi^{\prime} & =v \\
0 & =f(q, \varphi, i, v, t),
\end{aligned}
$$

where $f$ captures Kirchhoff laws but also the constitutive relations of all circuit devices. Aside from the different circuit topologies, reflected in the form of the loop and cutset matrices $B, D$ arising in item (b), the differences between circuit families come from the devices' characteristics referred to in (c).

Two-variable characteristics. In classical circuit theory, every characteristic within the map $f$ in (1c) just involves two out of the four variables mentioned above. Disregarding the memristor, these two-variable characteristics relate current and voltage in resistors and controlled sources, charge and voltage in capacitors, and flux and current in inductors; in general, these relations may be nonlinear and/or involve time explicitly. The memory-resistor or memristor, whose existence was predicted by Leon Chua in 1971 [1] and which actually appeared at the nanometer scale in 2008 [2], completes the set of two-variable characteristics by relating charge and flux. Note that the pairs $q-i$ and $\varphi-v$ are linked as indicated in item (a) above. Other (so-called) mem-devices, such as memcapacitors and meminductors as defined in [3], [4], [5], are also characterized by a relation between two variables, in this case involving either $\sigma$ (the time integral of $q$ ) or $\rho$ (integral of $\varphi$ ).

Characteristics involving three or four variables. The first goal of this paper is to present a circuit-theoretic framework accommodating also characteristics which involve three or even all four fundamental circuit variables. The idea is that the components of $f$ modeling individual devices can take the general form $h(q, \varphi, i, v, t)=0$ (cf. Definition 1), and the actual appearance of $q, \varphi, i$ and/or $v$ defines the electrical nature of the device. Needless to say, two-variable characteristics fit in well, but we find those involving more variables of interest for at least the three reasons discussed below.

1. The explicit description of memory effects in memdevices, by nature, requires more than two variables. For instance, the original characteristic of a charge-controlled memristor reads as $\varphi=\phi(q)$, and by differentiation we arrive at the voltage-current relation

$$
v=M(q) i \text {. }
$$

Here $M(q)=\phi^{\prime}(q)$ is the memristance, which keeps track of the device history because it depends on the integral variable $q(t)=\int_{-\infty}^{t} i(\tau) d \tau$. The description (2) involves $v, i$ and $q$, this third variable introducing memory in the device. But we might also think of circuit elements 
governed by a fully nonlinear characteristic of the form $v=\eta(q, i)$, not arising as the differentiated form of a twovariable relation and which may model a more intricate dependence on the device history.

2. In this direction, characteristics involving more than two variables capture the fact that different electrical effects may coexist in a single device. For instance, as discussed in [6], memristive and capacitive effects are expected to coexist in metal-insulator-metal thin films with thickness between the nanometer and the micrometer scales. This should be modelled by a relation between charge, flux and voltage of the form $q=\omega(\varphi, v)$; in this regard, see also [5], [7].

3. Additionally, within a black-box modelling approach [8], in which the electrical characteristics of a circuit element are to be identified experimentally by external measures, it may simply happen that a given device does not admit a description in terms of just two variables. An example of a two-terminal not admitting a two-variable description will be discussed in Section II. Generally speaking, allowing for characteristics with more than two variables may provide simplified two-terminal descriptions of connections of basic devices.

By discussing all possible combinations of two, three or even four of the variables $q, \varphi, i, v$, from a theoretical point of view we provide an exhaustive account of what might be called first order circuit theory, within the setting defined by items (a), (b) and (c) on p. 1. Here the term "order" is used to mean differential order (cf. Section II) and, accordingly, the expression "first order circuit" reflects the fact that only the branch currents and voltages $i, v$ and their first integrals $q, \varphi$ are involved. The reader should not misunderstand our use of this expression with that in elementary circuit theory, referring to a circuit with only one reactive element. To avoid ambiguities we will often use the expression "first order mem-circuit" to mean a mem-circuit with differential order one.

A modeling framework for first order circuits is detailed in Section II. The taxonomy of first order devices will be organized around the notions of the differential and the state order of a device. We will use fully nonlinear characteristics for all devices and, in particular, it will be shown that when three-variable relations are linear in certain variables they amount to the memristors, memcapacitors and meminductors introduced by Chua and Di Ventra et al. in [1], [3], [4], [5]. We will also discuss fourvariable characteristics accounting for memristive effects depending on both the charge and the flux.

The second goal of this paper, tackled in Sections III and IV, is to address certain analytical and dynamical properties of the aforementioned mem-circuits. Specifically, we analyze in Section III the differential-algebraic models arising from the nodal analysis of nonlinear circuits including fully nonlinear memristors, memcapacitors and meminductors. Our attention in this regard will be mainly focused on the characterization of the index of these models (cf. [9], [10], [11], [12], [13], [14]). We will arrive at a general index characterization (cf. Theorems 1 and 2 in Section III) from which the results discussed in [14], [15], [16], [17], [18] can be derived as particular cases. We will also extend the notion of a topologically degenerate configuration to this broader setting. In Section IV, certain bifurcation phenomena stemming from the presence of manifolds of non-isolated equilibria will be explored for a family of nonlinear oscillators involving mem-devices; in particular, our analysis will explain and extend several results obtained by Itoh and Chua in [19].

\section{FIRst ORDER DEVICES}

Definition 1. A circuit device is said to have differential order one if it is defined by a $C^{1}$-characteristic of the form

$$
h(q, \varphi, i, v, t)=0
$$

where at least one of the partial derivatives $h_{q}, h_{\varphi}$ does not vanish identically.

Note that both $h_{q}$ and $h_{\varphi}$ may of course vanish for specific values of the circuit variables. Definition 1 is supported on the fact that when both $h_{q}$ and $h_{\varphi}$ vanish identically, the only branch variables involved are the current and/or the voltage. This amounts to what may be called (differential) order zero devices, not involving any dynamics. These are nonlinear resistors, and voltage and current sources. By a nonlinear resistor we mean any device relating current and voltage in an algebraic (i.e., non-differential) manner, such as a diode.

The use of the term "order" is worth a digression. This term has different senses in mathematics, and at least two apply in our context. On the one hand it means the order of derivation of a system of differential equations; in this sense, Newton's law yields a second order system. We will use the expression differential order to mean this. On the other hand, "order" is also used to mean the number of dynamic variables in a system of differential equations; the term is often used in this sense in circuit theory, to refer to the number of state variables associated with a given device. We will describe the latter as the state order of a device. Throughout the document and when no label is used, by "order" we refer to the differential order; e.g. a first order device is a device with differential order one. Note that both concepts are certainly related, since any system with differential order higher than one can be recast as a first order one by introducing additional variables.

In practice, at least two of the derivatives $h_{q}, h_{\varphi}, h_{i}$, $h_{v}$ do not vanish identically in first order devices. It is also worth noting that in Definition 1 we implicitly assume the arguments of $h$ to be one-dimensional, hence describing a one-port (two-terminal) device; however, by allowing $q, \varphi, i$ and $v$ to take vector values, this definition easily accommodates multiport and multiterminal devices, accounting for coupling effects. In this case the nonvanishing requirement on $h_{q}$ and/or $h_{\varphi}$ must be replaced by the non-singularity of the corresponding matrices of 
partial derivatives. This remark will apply throughout the paper, often without explicit mention.

\section{A. $q$ - and $\varphi$-memristors}

The electromagnetic relations $q^{\prime}=i$ and $\varphi^{\prime}=v$ link the pairs of fundamental circuit variables $q-i$ and $\varphi-v$. First order devices involving the other combinations of two out of the four variables $q, \varphi, i, v$ are the capacitor, relating $q$ and $v$, the inductor, which involves $\varphi$ and $i$, and the memristor, whose characteristic relates $q$ and $\varphi$. Even though both $q$ and $\varphi$ arise in the characteristic of memristors, these variables are linked together and therefore only one of them introduces a dynamical degree of freedom. For this reason, it will be preferred to get rid of either the flux or the charge by means of a relation formulated in terms of the other three variables, as detailed below. This way, not only capacitors and inductors but also $q$ - and $\varphi$-memristors will have state order one.

Definition 2. A q-memristor is a device with differential order one, governed by the relations

$$
\begin{aligned}
q^{\prime} & =i \\
v & =\eta(q, i, t),
\end{aligned}
$$

where $\eta$ is a $C^{1}$-map for which neither of the derivatives $\eta_{q}, \eta_{i}$ vanishes identically.

For fully nonlinear memristors of the form (4) the incremental memristance is defined as the derivative $\eta_{i}(q, i, t)$ [20]. The identity $q(t)=\int_{-\infty}^{t} i(\tau) d \tau$ shows that this relation keeps track of the device history. The device is called strictly locally passive if $\eta_{i}(q, i, t)>0$ for all $(q, i, t)$ (or if the matrix $\eta_{i}$ is positive definite in multiports). The requirement that $\eta_{i}$ does not vanish identically distinguishes the device from a nonlinear capacitor. In turn, the non-vanishing condition on $\eta_{q}$ makes this device actually different from a nonlinear, current-controlled resistor. Note also that the fully nonlinear form (4) makes it possible to accommodate devices displaying memristive effects but whose characteristic does not arise as the time derivative of a $\varphi-q$ relation, contrary to Chua's memristor.

Definition 3. $A \varphi$-memristor is a device with differential order one, governed by

$$
\begin{aligned}
\varphi^{\prime} & =v \\
i & =\zeta(\varphi, v, t),
\end{aligned}
$$

where $\zeta$ is a $C^{1}$-map such that neither of the derivatives $\zeta_{\varphi}, \zeta_{v}$ vanishes identically.

The incremental memductance is the derivative $\zeta_{v}(\varphi, v, t)$, and the device is said to be strictly locally passive if this derivative is always positive. Again, the non-vanishing requirements on $\zeta_{\varphi}$ and $\zeta_{v}$ make this device different from a voltage-controlled resistor and an inductor, respectively.
Chua's memristors. When $\eta$ in (4b) is time-invariant and linear in $i$, we get Chua's characteristic $v=M(q) i$, where $M(q)$ is the memristance [1]; the dependence on $q$ led Chua to propose the name memory-resistor, or memristor for short. In the literature, this device is said to be currentcontrolled but also charge-controlled, because of the form of the map $\varphi=\phi(q)$ whose time derivative yields the voltage-current relation $v=M(q) i$. Analogously, a timeinvariant $\varphi$-memristor for which (5b) is linear in $v$ (hence reading as $i=W(\varphi) v$, coming from a charge-flux relation $q=\xi(\varphi))$ amounts to Chua's flux-controlled memristor [1], $W(\varphi)$ being the memductance.

Applications. The applications of memristors in the design of non-volatile memories, signal processing, adaptive and learning systems, reconfigurable nanoelectronics, etc., indicate that these devices will play a very significant role in electronics in the near future, specially at the nanometer scale. Many applications are reported in the literature; cf. [7], [16], [19], [21], [22], [23], [24], [25], [26], [27], [28], [29], [30], [31]. HP has announced that commercial memory chips based on the memristor will be released in 2013 [32]. Recent applications are reported on pattern recognition [33] and on neural and quantum computation [34], [35].

A two-terminal without a two-variable description. As indicated in the Introduction, a reason to consider descriptions involving more than two variables comes from the black-box approach to circuit modeling (cf. [8]). It may simply happen that a device does not admit a two-variable description; in that case, any effort to identify by external measures a characteristic involving only two variables and providing an accurate model of the device would be hopeless or lead to erroneous results. An example is presented below.

Consider the parallel connection of a Chua's fluxcontrolled memristor and a voltage-controlled resistor, as depicted in Fig. 1. This connection arises for instance in accurate models of the Josephson junction, as detailed later.

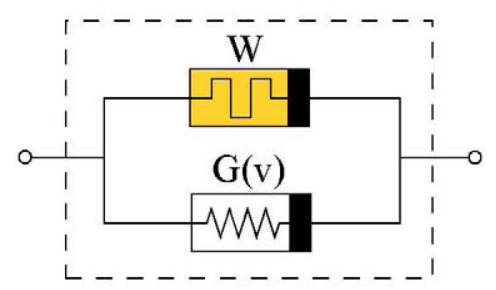

Fig. 1. A two-terminal not admitting a two-variable description.

The memristor is assumed to be governed by a chargeflux relation $q=\xi(\varphi)$, which induces a current-voltage relation of the form $i_{1}=W(\varphi) v$, with $W(\varphi)=\xi^{\prime}(\varphi)$. Assume first that the resistor is a linear one. In this case we have $i_{2}=G v$ for a given constant $G$. The parallel 
connection of both devices is then governed by the threevariable description $i=(W(\varphi)+G) v$, but also admits the two-variable characteristic $q=\xi(\varphi)+G \varphi$.

By contrast, suppose that the resistor exhibits a nonlinear effect which makes the conductance $G$ depend on $v$. Now the three-variable description of the device reads as

$$
i=(W(\varphi)+G(v)) v=K(\varphi, v) v
$$

and it is not difficult to see that the connection does not admit a two-variable characteristic. Note, indeed, that any such description $q=g(\varphi)$ would necessarily yield by differentiation a relation $i=g^{\prime}(\varphi) v$ which is linear in $v$, against the hypothesis that $G$ (and hence $K$ ) in (6) actually depends on $v$. Therefore, any attempt to describe Fig. 1 in terms of a charge-flux (or any other two-variable) characteristic would fail in the presence of a nonlinearity on the resistor making the conductance dependent on $v$; in this case, a three-variable description is mandatory.

\section{B. Fully nonlinear memcapacitors}

The remaining characteristics involving three out of the four variables $q, \varphi, i, v$ naturally lead to the voltagecontrolled memcapacitors and current-controlled meminductor discussed below. Both devices will have state order two. We consider fully nonlinear characteristics for memcapacitors and meminductors; when the maps $\omega$ and $\theta$ below are time-independent and linear in $v$ and $i$, respectively, we will get the circuit elements introduced by Di Ventra et al. in [5].

Definition 4. $A$ voltage-controlled memcapacitor is a device with differential order one, defined by

$$
\begin{aligned}
q^{\prime} & =i \\
\varphi^{\prime} & =v \\
q & =\omega(\varphi, v, t),
\end{aligned}
$$

where $\omega$ is a $C^{1}$-map and neither of the derivatives $\omega_{\varphi}$, $\omega_{v}$ vanishes identically.

Characteristics of the form (7c) model the coexistence of memristive and capacitive effects; in a timeinvariant setting, such a characteristic arises for instance in metal-insulator-metal films having thickness between the nanometer scale, where a $q-\varphi$ relation is dominant, and the micrometer scale, being governed by a $q-v$ relation [6].

For an arbitrary $C^{1}$-map $\omega$, the incremental memcapacitance $C_{\mathrm{m}}$ is defined as the derivative $\omega_{v}$ and, in general, depends on $(\varphi, v, t)$. Such a device need not come from a $\sigma-\varphi$ relation, and the requirement that neither $\omega_{\varphi}$ nor $\omega_{v}$ vanishes identically makes it actually different from a capacitor or a memristor, respectively.

An instance of a fully nonlinear memcapacitor arising in a Josephson junction model can be found below. Fully nonlinear memcapacitors will also arise in the nonlinear oscillators analyzed in Section IV.
Example: equivalent model of a Josephson junction. Our next example illustrates how fully nonlinear characteristics allow for simplified device descriptions. Specifically, we will provide an accurate description of a Josephson junction by means of a fully nonlinear memcapacitor, which accounts for all parasitic effects, connected in parallel to a nonlinear inductor. Josephson junctions are used e.g. in the design of quantum bits, such as the phase qubit, defined by a current-biased junction [36], or the charge qubit analyzed in Section III.
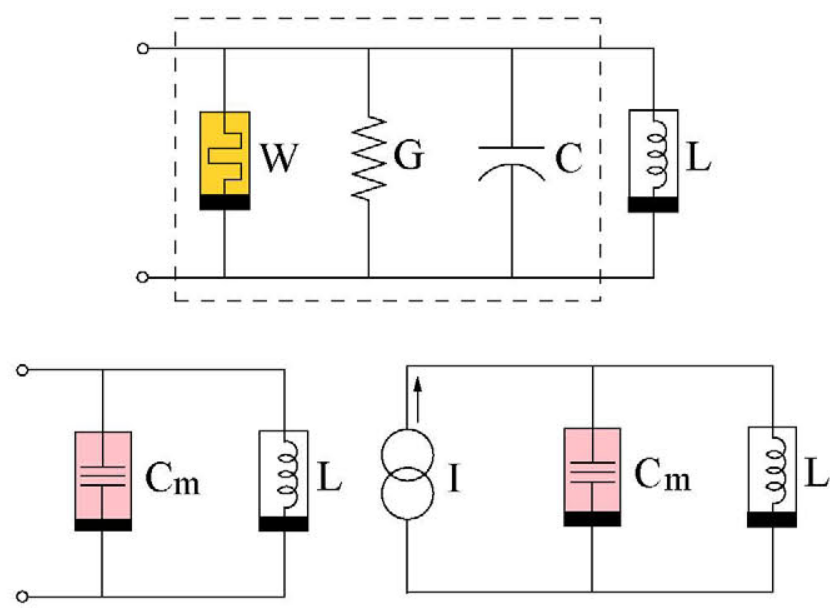

Fig. 2. Top: Josephson junction. Bottom: (Left) Equivalent circuit with a fully nonlinear memcapacitor. (Right) Phase qubit.

As detailed in [8], [37], realistic models of a Josephson junction should take into account not only the usual nonlinear inductive relation $i_{l}=I_{0} \sin \left(k_{0} \varphi_{l}\right)$ for certain physical constants $I_{0}, k_{0}$ (see e.g. [38]), but also the presence of memristive, resistive and capacitive effects; an accurate equivalent circuit of the Josephson junction is defined by the parallel connection of these four elements (cf. [8], [37]), as depicted on top of Fig. 2.

The device on the left is a $\varphi$-memristor of Chua type, which as reported in [8], [37] captures the presence of a small current component $i_{w}=G_{1} \cos \left(k_{1} \varphi_{w}\right) v$, for certain constants $G_{1}, k_{1}$; here $v$ is the port voltage.

A linear resistor and a linear capacitor in parallel are also present in the description provided in [8], [37], being denoted in the figure by $G, C$, respectively. These elements are defined by the relations $i_{g}=G v$ and $q_{c}=C v$.

Now, the parallel connection of the memristor and the resistor is obviously governed by the current-voltage relation $i_{w g}=G_{1} \cos \left(k_{1} \varphi_{w}\right) v+G v$ or, equivalently, by a charge-flux characteristic of the form

$$
q_{w g}=\left(G_{1} / k_{1}\right) \sin \left(k_{1} \varphi_{w}\right)+G \varphi_{w},
$$

where we use the fact that the memristor flux is the timeintegral of the port voltage $v$. The expression depicted in (8) shows that the parallel connection of the memristor and the resistor is itself a $\varphi$-memristor.

In turn, the parallel connection of the original memristor, the resistor and the capacitor can be described as 
a single device by setting $q=q_{w g}+q_{c}, \varphi=\varphi_{w}$. Indeed, denoting by $i$ the sum of the currents through the memristor, the resistor and the capacitor, we get

$$
q=\left(G_{1} / k_{1}\right) \sin \left(k_{1} \varphi\right)+G \varphi+C v,
$$

together with $q^{\prime}=i, \varphi^{\prime}=v$. This describes a timeinvariant, voltage-controlled memcapacitor for which the constitutive relation in (7c) takes the specific form depicted in (9). The corresponding equivalent circuit for the Josephson junction, together with the equivalent circuit for a phase qubit, is displayed at the bottom of Fig. 2 .

\section{Fully nonlinear meminductors}

Definition 5. A current-controlled meminductor is a device with differential order one, governed by

$$
\begin{aligned}
q^{\prime} & =i \\
\varphi^{\prime} & =v \\
\varphi & =\theta(q, i, t),
\end{aligned}
$$

where $\theta$ is a $C^{1}$-map for which neither of the derivatives $\theta_{q}, \theta_{i}$ vanishes identically.

In actual applications, meminductors arise e.g. in the design of generalized tank circuits (cf. [35]). In general, the derivative $\theta_{i}(q, i, t)$ is the incremental meminductance. The non-vanishing of this derivative makes the meminductor different from a memristor and, similarly, the assumption that the partial derivative $\theta_{q}$ does not vanish identically makes the device different from an inductor.

The appearance of both $q$ and $\varphi$ in the characteristics (7c) and (10c) imply that the time derivatives of two variables must be present in the dynamical description of memcapacitors and meminductors and, therefore, that these devices have state order two; this is an important difference with capacitors, inductors and $q$ - and $\varphi$ memristors, for which one dynamic variable suffices to describe the device behavior.

Memcapacitors and meminductors of Di Ventra et al. When $\omega$ in $(7 \mathrm{c})$ is time-invariant and linear in $v$, this relation reads as

$$
q=C_{\mathrm{m}}(\varphi) v
$$

where $C_{\mathrm{m}}$ is the memcapacitance [3], [4], [5]. The distinct feature of this device is that the memcapacitance depends on the state variable $\varphi(t)=\int_{-\infty}^{t} v(\tau) d \tau$, so that the relation $q(t)=C_{\mathrm{m}}\left(\int_{-\infty}^{t} v(\tau) d \tau\right) v(t)$ reflects the device history. Be aware of the circuit-theoretic meaning of this state variable, in contrast to memcapacitive systems (see [3], [4], [5]). It is also worth mentioning that the relation $q=C_{\mathrm{m}}(\varphi) v$ arises as the derivative of a characteristic $\sigma=\mu(\varphi)$, where $\sigma$ is the time integral of $q$. This yields $\sigma^{\prime}=q=\mu^{\prime}(\varphi) \varphi^{\prime}=C_{\mathrm{m}}(\varphi) v$. Notably, the device can be described without recourse to the (second order) variable $\sigma$ (cf. (7)), in contrast to charge-controlled memcapacitors (cf. [5]).
Similarly, when the map $\theta$ in (10c) is linear in $i$ and does not depend on $t$, we get the characteristic

$$
\varphi=L_{\mathrm{m}}(q) i
$$

considered by Chua and by Di Ventra et al. in [3], [4], [5]. Such a characteristic can be obtained as the time derivative of a $C^{1}$-relation $\rho=\kappa(q)$, where $\rho$ is the time integral of $\varphi$; note however that the description (10) does not involve $\rho$. Now $L_{\mathrm{m}}$ is the meminductance.

\section{Characteristics involving four variables}

In light of the characteristics (4b), (5b), (7c) and (10c), from a mathematical point of view it is somehow natural to complete the picture by considering a relation which involves all four variables $q, \varphi, i$ and $v$. We present below two different settings (dual to each other) in which these four variables may actually arise.

These characteristics may account for physical devices in which memory effects of different nature coexist. The simultaneous appearance of memristive, memcapacitive and/or meminductive phenomena has been discussed by different authors. For instance, the coexistence of memristive and memcapacitive effects has been reported to follow from the formation of local dipoles in nanoscale resistors [5], and as indicated above arises also in metal-insulatormetal thin films with thickness between the nanometer and the micrometer scales [6]. Capacitive and memristive effects coexist with the nonlinear inductive nature of a Josephson junction in accurate models of this device, as discussed above. From a modeling point of view, these characteristics also allow for simplified descriptions of combinations of more basic devices.

Consider, in particular, a characteristic of the form

$$
v=\psi(q, \varphi, i, t),
$$

where $\psi$ is a $C^{1}$-map and none of the derivatives $\psi_{q}$, $\psi_{\varphi}, \psi_{i}$ vanishes identically. Such a characteristic may be understood to define a current-controlled hybrid memristor, note that if (13) is time-independent and linear in $i$, this relation takes the form $v=M_{\mathrm{h}}(q, \varphi) i$, providing an analog of Chua's memristor in which the so-called hybrid memristance $M_{\mathrm{h}}(q, \varphi)$ would depend on both the charge and the flux; both variables introduce memory effects on the device, because of the relations $q(t)=\int_{-\infty}^{t} i(\tau) d \tau$, $\varphi(t)=\int_{-\infty}^{t} v(\tau) d \tau$. An example is presented below.

Analogously, a voltage-controlled hybrid memristor might be understood to be defined by a characteristic of the form

$$
i=\Lambda(q, \varphi, v, t),
$$

where $\Lambda$ is a $C^{1}$-map for which none of the derivatives $\Lambda_{q}, \Lambda_{\varphi}, \Lambda_{v}$ vanishes identically. Cases in which $\Lambda$ is linear in $v$ and time-invariant yield $i=W_{\mathrm{h}}(q, \varphi) v$, where $W_{\mathrm{h}}$ would now be the hybrid memductance.

The non-vanishing requirements on the derivatives within (13), (14) distinguish these devices from the $q$ - 
and $\varphi$-memristors, memcapacitors and meminductors discussed above. Actually, the non-vanishing of these derivatives implies that, at least locally, both the charge and the flux can be written in terms of the remaining circuit variables. Focusing the attention on the current-controlled case, the fact that $\psi_{q} \neq 0$ makes it possible to recast (13), via the implicit function theorem, as

$$
q=\alpha(\varphi, i, v, t)
$$

and the device exhibits a (generalized) memcapacitance $\alpha_{v}(\varphi, i, v, t)=\psi_{q}^{-1}(\alpha(\varphi, i, v, t), \varphi, i, t)$. Similarly, the non-zero nature of the partial derivative $\psi_{\varphi}$ yields

$$
\varphi=\beta(q, i, v, t),
$$

for some locally defined map $\beta$, the (generalized) meminductance being defined by the derivative $\beta_{i}(q, i, v, t)=$ $-\psi_{\varphi}^{-1}(q, \beta(q, i, v, t), i, t) \psi_{i}(q, \beta(q, i, v, t), i, t)$, as a consequence of the implicit function theorem. Similar remarks apply to voltage-controlled hybrid memristors.

Noteworthy, disregarding the non-vanishing requirements on the derivatives, the relations (13), (14), (15) and (16) account for the characteristics of all previous devices.

Example: hybrid description of series and parallel connections of Chua's memristors. Suppose that the resistor within the Josephson junction model depicted in Fig. 2 exhibits a memristive effect which makes the conductance $G$ depend on $q$. As detailed in what follows, the resulting parallel connection of a flux-controlled and a charge-controlled memristor would yield a simple instance of a hybrid memristor.

In broader generality, consider both the series and the parallel connection of a charge-controlled and a fluxcontrolled memristor of Chua type, as displayed in Fig. 3. The charge-controlled memristor and the flux-controlled one are painted in green and yellow, respectively. As before, the goal is to provide a dynamical description of each connection as a two-terminal device in terms of a single set of variables $q, \varphi, i, v$.

In both cases, the subscripts 1 and 2 will correspond to variables associated with the charge- and the fluxcontrolled memristor, respectively. The charge-controlled memristor is assumed to be governed by a relation of the form $\varphi_{1}=\phi\left(q_{1}\right)$, with memristance $M\left(q_{1}\right)$, and the flux-controlled one is defined by $q_{2}=\xi\left(\varphi_{2}\right)$, with memductance $W\left(\varphi_{2}\right)$. Within the series connection on top of Fig. 3 (resp. the parallel connection at the bottom), the memductance $W\left(\varphi_{2}\right)$ (resp. the memristance $M\left(q_{1}\right)$ ) is assumed not to vanish.

In the series connection on top of Fig. 3, elementary circuit theory yields $i=i_{1}=i_{2}, v=v_{1}+v_{2}$. In order to arrive at a dynamical description in terms of $i, v$ and single variables $q$, $\varphi$, we set $q=q_{1}$ and $\varphi=\varphi_{1}+\varphi_{2}$. Obviously, this yields the relations $q^{\prime}=i$ and $\varphi^{\prime}=v$ but, more important, allows for the description of the voltagecurrent relation in the form $v=M\left(q_{1}\right) i+\left(W\left(\varphi_{2}\right)\right)^{-1} i=$
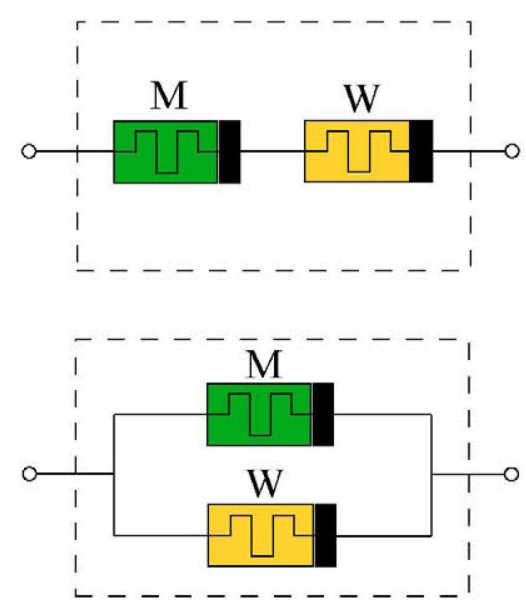

Fig. 3. Series and parallel connection of Chua's memristors.

$\left[M(q)+(W(\varphi-\phi(q)))^{-1}\right] i$. This corresponds to a currentcontrolled hybrid memristor for which the characteristic is linear in $i$, the hybrid memristance being

$$
M_{\mathrm{h}}(q, \varphi)=M(q)+(W(\varphi-\phi(q)))^{-1} .
$$

The physical meaning of the variable $q$ is worth some additional remarks. Because of the identities $q_{1}^{\prime}=i_{1}=$ $i_{2}=q_{2}^{\prime}$, the charges $q_{1}$ and $q_{2}$ differ in a constant which would be fixed by the initial conditions in Chua's memristors. In turn, $q$ is defined up to a constant, and therefore can be understood to describe any of both charges except for a fixed quantity. Mathematically, setting $q=q_{1}+k$ for any real constant $k$, we would get a dynamical description of the device which, except for an affine change of coordinates, amounts to the one above; the description above assumes $k=0$ so that $q$ actually equals the charge $q_{1}$.

It is also worth noting that if $M(q)+(W(\varphi-\phi(q)))^{-1}$ does not vanish for all values of $q, \varphi$, the device also admits a voltage-controlled description. This would be the case, in particular, if the original Chua memristors are strictly locally passive (i.e. if $M>0, W>0$ everywhere); in this case the hybrid memristor would itself be strictly locally passive since $M_{\mathrm{h}}$ would be positive.

Setting $q=q_{1}+q_{2}, \varphi=\varphi_{2}$, the reader can proceed analogously in order to describe the parallel configuration at the bottom of Fig. 3 as a voltage-controlled hybrid memristor, with a characteristic linear in $v$ and hybrid memductance $W_{\mathrm{h}}(q, \varphi)=(M(q-\xi(\varphi)))^{-1}+W(\varphi)$.

This shows that, even in simple examples, these devices pose interesting problems from the modeling perspective. The scope of these four-variable characteristics goes however beyond this type of examples. Such characteristics are aimed at modeling devices which are not reducible to a connection of $q$ - and $\varphi$-memristors, and which may capture the coexistence of different memory effects. In such devices the characteristics need not be linear in $i$ and $v$, and the charge and the flux within the memristance or memductance may interact in more intricate ways. 


\section{NODAL ANALYSIS OF FIRST ORDER MEM-CIRCUITS}

From a computational point of view, models of the form (1) offer some difficulties for numerical simulation. This is due to the fact that an automatic computation of the loop and cutset matrices $B, D$ is difficult to perform in practice, specially in high scale integration circuits. For this reason, it is often preferred to describe the circuit equations using nodal analysis (cf. subsection III-A). This is the case in most circuit simulation programs, notably in SPICE and its commercial variants, which set up the circuit equations using Modified Nodal Analysis (MNA) [14], [15], [17], [18], [39], [40], [41], [42], [43].

As detailed below, the models arising from nodal analysis naturally take the form of a differential-algebraic equation (DAE) [9], [10], [11], [12], [13], [14]. The main problem in the analysis of such differential-algebraic models is the characterization of the index, a concept which, roughly speaking, measures the numerical difficulties faced in simulation. Index one and index two systems require specific numerical techniques, and because of this it is important to characterize the circuit configurations which lead to models with these indices. Therefore, we undertake in subsections III-B and III-C the index analysis of nodal models of first order mem-circuits, using the tractability index framework [12], [18], [44] and extending the results discussed in [14], [15], [16], [17] to fully nonlinear circuits with memristors, voltage-controlled memcapacitors and current-controlled meminductors.

Index one circuit configurations are also important regarding the order of complexity and the state-space formulation problem [14], [45], [46], [47], [48], [49], [50]. In index one systems, all the dynamic variables of the different devices contribute to the state dimension of the problem; more precisely, in index one cases the state dimension (also called the order of complexity) of a first order circuit equals the sum of the state orders of the devices with differential order one. By contrast, in higher index problems which arise from so-called topologically degenerate configurations the feasible values for these dynamic variables are restricted by algebraic (nondifferential) constraints. In this regard, we introduce below the notion of a topologically degenerate configuration for circuits including memcapacitors and meminductors, and show that these configurations lead to index two DAEs.

The model introduced in (17) will also pave the way for a discussion of some qualitative features of first order mem-circuits. Specifically, in subsection III-D we will discuss the existence of vanishing natural frequencies related to the presence of manifolds of non-isolated equilibria in mem-circuits. Related bifurcations will be addressed in the nonlinear oscillators considered in Section IV.

\section{A. The nodal model}

Nodal models are based on the systematic use of the reduced incidence matrix $A$, which for a connected circuit is defined as $A=\left(a_{i j}\right)$ with

$$
a_{i j}=\left\{\begin{aligned}
1 & \text { if branch } j \text { leaves node } i \\
-1 & \text { if branch } j \text { enters node } i \\
0 & \text { if branch } j \text { is not incident with node } i
\end{aligned}\right.
$$

for all nodes except for the reference one. In (17) this matrix will be partitioned by columns according to the electrical nature of the corresponding branches. In a timeinvariant setting, the nodal model then reads as

$$
\begin{aligned}
q_{0}^{\prime}= & i_{0} \\
\varphi_{1}^{\prime}= & A_{1}^{T} e \\
0= & A_{c} i_{c}+A_{m c} i_{m c}+A_{u} i_{u}+A_{l} i_{l}+A_{m l} i_{m l} \\
& +A_{g} \gamma_{g}\left(A_{g}^{T} e\right)+A_{w} \zeta\left(\varphi_{w}, A_{w}^{T} e\right)+A_{m} i_{m} \\
& +A_{j} i_{s}(t) \\
0= & q_{c}-\gamma_{c}\left(A_{c}^{T} e\right) \\
0= & q_{m c}-\omega\left(\varphi_{m c}, A_{m c}^{T} e\right) \\
0= & v_{s}(t)-A_{u}^{T} e \\
0= & \varphi_{l}-\gamma_{l}\left(i_{l}\right) \\
0= & \varphi_{m l}-\theta\left(q_{m l}, i_{m l}\right) \\
0= & \eta\left(q_{m}, i_{m}\right)-A_{m}^{T} e
\end{aligned}
$$

where, for the sake of notational simplicity, we are denoting $q_{0}=\left(q_{c}, q_{m c}, q_{m}, q_{m l}\right), i_{0}=\left(i_{c}, i_{m c}, i_{m}, i_{m l}\right)$, $\varphi_{1}=\left(\varphi_{l}, \varphi_{m l}, \varphi_{w}, \varphi_{m c}\right), A_{1}=\left(A_{l} A_{m l} A_{w} A_{m c}\right)$.

The key features of this model are the use of node potentials $e$ and the description of Kirchhoff laws as $v=A^{T} e, A i=0$. The former is used to eliminate branch voltages throughout the model. The latter is depicted in (17c), where we eliminate the branch currents $i_{g}, i_{w}, i_{j}$ of resistors, $\varphi$-memristors and current sources by means of the maps $\gamma_{g}, \zeta, i_{s}(t)$ respectively. The charge-flux formalism yields (17a) and (17b), which account for the relations $q^{\prime}=i, \varphi^{\prime}=v$, whereas (17d)-(17i) capture the characteristics of capacitors, memcapacitors, voltage sources, inductors, meminductors and $q$-memristors; the subscripts corresponding to these devices are $c, m c, u, l$, $m l$ and $m$, respectively.

For simplicity we do not include devices defined by four-variable characteristics, although some remarks in this direction can be found at the end of subsection III-C. Capacitors and inductors are assumed to be voltage- and current-controlled, respectively (cf. the maps $\gamma_{c}$ and $\gamma_{l}$ in $(17 \mathrm{~d})$ and $(17 \mathrm{~g}))$. Note that resistors are assumed to be voltage-controlled, although the results can be extended without difficulty to models including also currentcontrolled ones. The functions $i_{s}(t)$ and $v_{s}(t)$ are the excitations in current and voltage sources, respectively. The maps $\eta, \omega, \theta$, are those arising in the characteristics $(4 b),(7 c)$, and $(10 \mathrm{c})$, except for the fact that they account for the whole sets of $q$-memristors, memcapacitors and meminductors, and hence need not be scalar; the same holds, of course, for $\varphi$-memristors and the map $\zeta$.

For later use, denote by $G, C, L, M, W, C_{\mathrm{m}}$ and $L_{\mathrm{m}}$ the incremental conductance, capacitance, inductance, 
memristance, memductance, memcapacitance and meminductance matrices, defined by the derivatives $\gamma_{g}^{\prime}, \gamma_{c}^{\prime}, \gamma_{l}^{\prime}$, $\eta_{i_{m}}, \zeta_{v_{w}}, \omega_{v_{m c}}, \theta_{i_{m l} l}$, respectively. These matrices need not be diagonal, meaning that full coupling is allowed within each of these sets of devices.

\section{B. Topologically nondegenerate configurations and index one models}

Semiexplicit differential-algebraic equations are defined by a system of the form

$$
\begin{aligned}
x^{\prime} & =f(x, y, t) \\
0 & =g(x, y, t),
\end{aligned}
$$

where $x \in \mathbb{R}^{r}$ stands for the dynamic variables, $y \in \mathbb{R}^{p}$ denotes the algebraic ones, $f \in C^{1}\left(\mathbb{R}^{r+p+1}, \mathbb{R}^{r}\right)$, and $g \in C^{1}\left(\mathbb{R}^{r+p+1}, \mathbb{R}^{p}\right)$. The DAE (18) is said to be index one around a given $\left(x^{*}, y^{*}, t^{*}\right)$ satisfying $(18 \mathrm{~b})$ if the matrix of partial derivatives $g_{y}\left(x^{*}, y^{*}, t^{*}\right)$ is invertible. Often, this non-singularity requirement holds everywhere. Detailed discussions about the different index notions can be found in [9], [10], [11], [12], [13], [14].

The nodal model (17) has a semiexplicit form, the set of dynamic variables being $q_{0}=\left(q_{c}, q_{m c}, q_{m}, q_{m l}\right), \varphi_{1}=$ $\left(\varphi_{l}, \varphi_{m l}, \varphi_{w}, \varphi_{m c}\right)$, whereas the algebraic ones are

$$
e, i_{c}, i_{m c}, i_{u}, i_{l}, i_{m l}, i_{m}
$$

We address below the characterization of index one configurations for (17), under the assumption that certain circuit matrices are positive definite; recall that a given matrix $K$ is positive definite if $u^{T} K u>0$ for any non-vanishing real vector $u$, and that this notion expresses mathematically a strict passivity requirement on the corresponding devices. The proof proceeds by showing how the non-singularity of the matrix defining index one configurations can be reduced to a form already analyzed in the context of nonlinear circuits without mem-devices in [14]. Theorem 1 actually motivates the following definition.

Definition 6. A first order mem-circuit is said to be topologically nondegenerate if it does not display either loops defined by voltage sources, capacitors and/or memcapacitors, or cutsets composed of current sources, inductors and/or meminductors.

This extends a well-known notion for RLC circuits, for which topologically nondegenerate configurations preclude loops defined by voltage sources and/or capacitors, and cutsets composed of current sources and/or inductors. Stemming from the work of Bashkow [45] in the classical RLC setting, this provides a way to formulate a state space model of the circuit dynamics by means on the notion of a proper tree.

Theorem 1. Assume that the capacitance $C$, the memcapacitance $C_{\mathrm{m}}$, the inductance $L$ and the meminductance $L_{\mathrm{m}}$ are non-singular matrices, and that the conductance $G$, the memristance $M$, and the memductance $W$ are positive definite.
Then the model (17) is index one if and only if the circuit is topologically nondegenerate.

Proof. The matrix of partial derivatives of the right-hand side of (17) with respect to all variables but time, to be denoted by $F$, has the form

$$
F=\left(\begin{array}{cc}
0 & F_{12} \\
F_{21} & F_{22}
\end{array}\right)
$$

where the block $F_{22}$ stands for the partial derivatives of the restrictions (17c)-(17i) with respect to the algebraic variables (19). The non-singularity of $F_{22}$ characterizes index one configurations, and this matrix has the expression depicted at the top of the next page. Using the nonsingularity of $C, C_{\mathrm{m}}, L, L_{\mathrm{m}}$ and by means of a Schur reduction [14], [51], the problem amounts to analyzing the non-singularity of the matrix $J$ displayed right after $F_{22}$. This matrix has the structure arising in $[14$, Theorem 5.1(1)] (cf. eq. (5.43) there); from this result it follows that, in the present setting, the non-singularity of this matrix relies on the absence of loops composed of voltage sources, capacitors and/or memcapacitors and cutsets defined by current sources, inductors and/or meminductors, as we aimed to show.

\section{Topological degeneracies: Index two}

In presence of the topologically degenerate configurations discussed above (i.e. loops defined by voltage sources, capacitors and/or memcapacitors, or cutsets composed of current sources, inductors and/or meminductors), the index one condition for the nodal system (17) fails. In this situation, for both analytical and numerical purposes it is important to characterize whether the model is index two or not. The index two notion for a DAE is more intricate than the index one concept introduced above. Again, the reader is referred to [9], [10], [11], [12], [13], [14] for different approaches to the index notion.

In particular, the tractability index notion, together with the projector-based framework supported on it [12], [14], [18], [44], has been proved to be a valuable tool in circuit simulation [14], [15], [17], [18], [40], [44]. In order to introduce this notion, we look at (17) as a semilinear problem of the form

$$
E z^{\prime}=h(z, t),
$$

where $E$ is a block-diagonal matrix block-diag $\{I, 0\}$, and $z$ joins together the $x$ - and $y$-variables in (18). Consider the matrix pencil $\lambda E-F, F$ being the matrix of partial derivatives $h_{z}$. As detailed in the references above, the pencil has tractability index one if $E_{1}=E-F Q$ is nonsingular, $Q$ being a projector onto ker $E$. In turn, if $E_{1}$ is singular, we let $Q_{1}$ be any projector onto $\operatorname{ker} E_{1}$, and the pencil is said to have tractability index two if $E_{2}=$ $E_{1}-F_{1} Q_{1}$ is non-singular, where $F_{1}=F(I-Q)$.

Iteratively, this approach provides a general index notion which can be shown to equal the Kronecker-Weierstrass 


$$
\begin{aligned}
& F_{22}=\left(\begin{array}{ccccccc}
A_{g} G A_{g}^{T}+A_{w} W A_{w}^{T} & A_{c} & A_{m c} & A_{u} & A_{l} & A_{m l} & A_{m} \\
-C A_{c}^{T} & 0 & 0 & 0 & 0 & 0 & 0 \\
-C_{\mathrm{m}} A_{m c}^{T} & 0 & 0 & 0 & 0 & 0 & 0 \\
-A_{u}^{T} & 0 & 0 & 0 & 0 & 0 & 0 \\
0 & 0 & 0 & 0 & -L & 0 & 0 \\
0 & 0 & 0 & 0 & 0 & -L_{\mathrm{m}} & 0 \\
-A_{m}^{T} & 0 & 0 & 0 & 0 & 0 & M
\end{array}\right), \\
& J=\left(\begin{array}{cccc}
A_{g} G A_{g}^{T}+A_{w} W A_{w}^{T}+A_{m} M^{-1} A_{m}^{T} & A_{c} & A_{m c} & A_{u} \\
-A_{c}^{T} & 0 & 0 & 0 \\
-A_{m c}^{T} & 0 & 0 & 0 \\
-A_{u}^{T} & 0 & 0 & 0
\end{array}\right)
\end{aligned}
$$

(or nilpotency) index of the pencil, being well-suited for computational purposes. Moreover, this concept can be extended to nonlinear and/or time-varying settings under suitable assumptions on the system operators; restricting the attention to DAEs of the form (21) in an index two context, these assumptions amount to requiring that $Q_{1}$ be a continuous projector onto the kernel of $E_{1}(z)$. Supported on these ideas, we show below that the nodal model (17) is indeed index two in the presence of degenerate configurations, under additional passivity requirements. Note that in the index two context the normal tree method of Bryant [46], [47] applies in order to derive a state space equation.

Theorem 2. Assume that the capacitance $C$, the memcapacitance $C_{\mathrm{m}}$, the inductance $L$, the meminductance $L_{\mathrm{m}}$, the conductance $G$, the memristance $M$, and the memductance $W$ are positive definite.

Then the nodal model (17) has tractability index two in the presence of topologically degenerate configurations.

Proof. As indicated above, the model (17) can be written in the form depicted in (21) with $E=$ block-diag $\{I, 0\}$ and $F$ being the matrix or partial derivatives displayed in (20). Letting $Q$ be a projector onto the kernel of $E$ with the structure block-diag $\{0, I\}$, we arrive at

$$
E_{1}=\left(\begin{array}{ll}
I & -F_{12} \\
0 & -F_{22}
\end{array}\right) .
$$

Note, incidentally, that this makes it clear why the (tractability) index one condition arising in Theorem 1 relies on the non-singularity of $F_{22}$. As indicated in the proof of Theorem 1, the presence of loops defined by voltage sources, capacitors and/or memcapacitors, and/or cutsets composed of current sources, inductors and/or meminductors makes $F_{22}$ (and hence $E_{1}$ ) a singular matrix.

In this setting, index two configurations can be examined using a projector $\bar{Q}$ onto

$$
\operatorname{ker}\left(A_{c} A_{m c} A_{u} A_{g} A_{w} A_{m}\right)^{T},
$$

being non-trivial in the presence of cutsets defined by inductors, meminductors and/or current sources, together with a projector

$$
\hat{Q}=\left(\begin{array}{lll}
\hat{Q}_{11} & \hat{Q}_{12} & \hat{Q}_{13} \\
\hat{Q}_{21} & \hat{Q}_{22} & \hat{Q}_{23} \\
\hat{Q}_{31} & \hat{Q}_{32} & \hat{Q}_{33}
\end{array}\right)
$$

onto $\operatorname{ker}\left(A_{c} A_{m c} A_{u}\right)$, which does not vanish in the presence of loops composed of capacitors, memcapacitors and/or voltage sources.

Omitting some technical details, the index two conditions can be checked to rely on the non-singularity of

$$
\left(\begin{array}{ccc}
A_{1} M_{1} A_{1}^{T}+A_{2} M_{2} A_{2}^{T} \bar{Q} & A_{3} & A_{4} \\
-M_{3} A_{3}^{T} & \tilde{Q} & \check{Q} \\
-A_{4}^{T} & 0 & 0
\end{array}\right)
$$

with

$$
\begin{aligned}
A_{1} & =\left(A_{g} A_{w} A_{m}\right) \\
M_{1} & =\operatorname{blockdiag}\left(G, W, M^{-1}\right) \\
A_{2} & =\left(A_{l} A_{m l}\right) \\
M_{2} & =\operatorname{blockdiag}\left(L^{-1}, L_{\mathrm{m}}^{-1}\right) \\
A_{3} & =\left(A_{c} A_{m c}\right) \\
M_{3} & =\operatorname{blockdiag}\left(C, C_{\mathrm{m}}\right) \\
A_{4} & =A_{u}
\end{aligned}
$$

and

$$
\tilde{Q}=\left(\begin{array}{ll}
\hat{Q}_{11} & \hat{Q}_{12} \\
\hat{Q}_{21} & \hat{Q}_{22}
\end{array}\right), \check{Q}=\left(\begin{array}{c}
\hat{Q}_{13} \\
\hat{Q}_{23}
\end{array}\right) .
$$

The form of the matrix depicted in (22) amounts to that arising in the index-two analysis of Augmented Nodal Analysis models of nonlinear circuits without memristive devices, which is proved in [14, Theorem 5.1(2)] to be non-singular provided that $M_{1}, M_{2}, M_{3}$ are positive definite. In this case, the definiteness of these matrices follows from the assumption that the circuit matrices are positive definite and the proof is complete.

In problems without memristive devices, Theorems 1 and 2 particularize to the results obtained in [14], [15], [17]. Our results also extend the characterization derived in 
[16] for cases including Chua's memristors and neither memcapacitors nor meminductors. Note that the scope of the general index characterization here presented covers $q$ - and $\varphi$-memristors, voltage-controlled memcapacitors and current-controlled meminductors, allowing for fully nonlinear characteristics in all of them. These results should be useful in future analytical or numerical studies of general first order mem-circuits.

Example: index change in a charge qubit. The circuit depicted in Fig. 4 is aimed at illustrating the index analysis presented above. It arises as a series connection of a voltage source, a capacitor and a Josephson junction, which defines a charge qubit [36]; in the equivalent circuit of the Josephson junction we accommodate parasitic effects of memristive, resistive and capacitive nature.

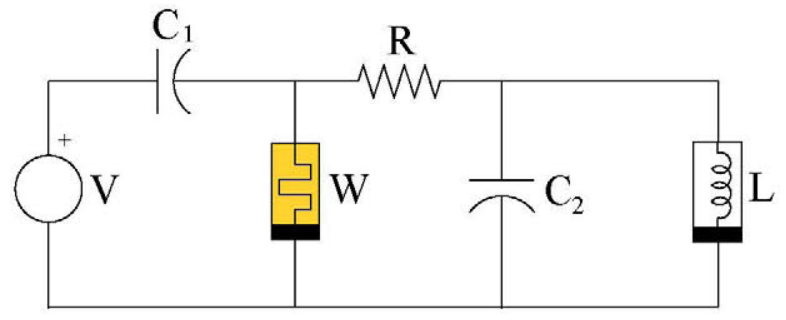

Fig. 4. Parasitic effects in a charge qubit.

A reduction of (17) modeling this circuit and retaining the index can be written, in a DC context, as

$$
\begin{aligned}
C_{1} e_{1}^{\prime} & =-i_{c_{1}} \\
C_{2} e_{2}^{\prime} & =-I_{0} \sin \left(k_{0} \varphi_{l}\right)+i_{c_{1}}-W\left(\varphi_{w}\right) e_{1} \\
\varphi_{w}^{\prime} & =e_{1} \\
\varphi_{l}^{\prime} & =e_{2} \\
0 & =e_{1}-e_{2}-R\left(i_{c_{1}}-W\left(\varphi_{w}\right) e_{1}\right),
\end{aligned}
$$

where $e_{1}$ and $e_{2}$ are the node potentials on top of the memristor and the capacitor $C_{2}$. Assume that all circuit parameters (except possibly the resistance $R$ ) are positive. It is not difficult to check that this model is index one provided that $R \neq 0$, and index two if $R=0$. This is a consequence of Theorems 1 and 2, more specifically of the absence of topologically degenerate configurations in the first case, and of the appearance in the second one of a VC-loop (defined by the voltage source and the two capacitors) after short-circuiting the resistor, that is, after making $R=0$.

The index change occurring when $R$ vanishes has interesting qualitative effects, and it is worth considering what happens when this parameter takes on negative values. Equilibria are defined by the vanishing of $e_{1}, e_{2}$ and $i_{c_{1}}$, together with the condition $k_{0} \varphi_{l}=m \pi$ for $m \in \mathbb{Z}$; to simplify matters, fix $\varphi_{l}=0$. Note that $\varphi_{w}$ does not enter these equilibrium conditions, meaning that the equilibrium locus is actually a one-dimensional manifold (find additional details in this regard in subsection III-D below). This implies, in particular, that one eigenvalue of the matrix pencil defining the linearization of (23) at equilibrium always vanishes. The remaining eigenvalues are given by the roots of the polynomial

$$
\begin{aligned}
R C_{1} C_{2} \lambda^{3}+\left(C_{1}+\right. & \left.C_{2}+R W C_{2}\right) \lambda^{2}+ \\
& +\left(R C_{1} I_{0} k_{0}+W\right) \lambda+(1+R W) I_{0} k_{0} .
\end{aligned}
$$

Akin to the results in [52], a singularity-induced bifurcation is expected to occur as $R$ crosses the origin. In more detail, one eigenvalue of the matrix pencil is expected to diverge through $\pm \infty$ as the resistance undergoes the value $R=0$, because of the failing of the index one condition at this point. This is actually the case; fixing, for simplicity, all circuit constants (except for $R$ ) at 1 , computer calculations show that one real eigenvalue jumps from large negative values (for small positive values of $R$ ) to large positive values (for small negative $R$ ); the remaining two eigenvalues are located at $-0.25 \pm 0.66 i$ at the bifurcation value, showing that a stability loss in the equilibrium curve occurs when $R$ becomes negative. It is worth emphasizing that this is a consequence of the index change displayed at $R=0$.

Circuits including devices with four-variable characteristics. In the model (17) and in the index analysis presented above we do not include devices defined by fourvariable characteristics for the sake of brevity. However, the index characterization presented in Theorems 1 and 2 applies also to circuits with such devices, as long as they are strictly locally passive.

More specifically, defining the incremental hybrid memristance $M_{\mathrm{h}}$ as the derivative $\psi_{i}(q, \varphi, i)$ ( $\psi$ standing for the characteristic defined in (13); we are assuming the devices to be autonomous), and the incremental hybrid memductance $W_{\mathrm{h}}$ as $\Lambda_{v}(q, \varphi, v)$ (cf. (14)), these circuit elements are said to be strictly locally passive if $M_{\mathrm{h}}$ and $W_{\mathrm{h}}$ are everywhere positive definite. Such devices do not interfere in the definition of a topologically degenerate configuration, and provided that they are strictly locally passive, the results stated in Theorems 1 and 2 still apply. Note that the proof proceeds with trivial modifications, associated with an additional term of the form $A_{h w} W_{\mathrm{h}} A_{h w}^{T}+A_{h m} M_{\mathrm{h}}^{-1} A_{h m}^{T}$ within the matrix $J$ above; in the index two case, the additional blocks $W_{\mathrm{h}}$ and $M_{\mathrm{h}}^{-1}$ arise in the block-diagonal matrix $M_{1}$, the reasoning being exactly the same. Details are left to the reader.

\section{Manifolds of equilibria}

The presence of non-isolated equilibria in the circuit of Fig. 4 actually owes to a general property of mem-circuits. Curves of equilibrium points have been already observed in circuits with memristors (see e.g. [19], [20], [24]) and we extend below some results in this regard to problems with memcapacitors and meminductors, in terms of the model (17). Some examples in Section IV will illustrate the discussion. This analysis will be performed under the 
zero-crossing conditions

$$
\zeta\left(\varphi_{w}, 0\right)=\eta\left(q_{m}, 0\right)=\omega\left(\varphi_{m c}, 0\right)=\theta\left(q_{m l}, 0\right)=0
$$

which mean that the vanishing of $v_{w}, i_{m}, v_{m c}$ and $i_{m l}$ implies that of $i_{w}, v_{m}, q_{m c}$ and $\varphi_{m l}$, respectively. Note that this holds in the setting of Chua [1] and Di Ventra et al. [5], in light of the relations $i_{w}=W(\varphi) v_{w}$, $v_{m}=M(q) i_{m}, q_{m c}=C_{\mathrm{m}}(\varphi) v_{m c}$ and $\varphi_{m l}=L_{\mathrm{m}}(q) i_{m l}$ assumed in their framework.

In a DC setting (that is, assuming $i_{s}=I, v_{s}=V$ to be constant) equilibria are defined by the vanishing of the right-hand side of (17). A careful inspection of this requirement under the zero-crossing conditions (24) shows that neither $\varphi_{w}, \varphi_{m c}$ nor $q_{m}, q_{m l}$ show up in the equilibrium conditions; this implies that any equilibrium of a mem-circuit is embedded into a manifold of equilibria whose dimension equals the total number of mem-devices (that is, $q$ - and $\varphi$-memristors, memcapacitors and meminductors). In a nonlinear context, these vanishing natural frequencies yield a zero eigenvalue whose geometric multiplicity at least equals the number of mem-devices.

On the other hand, it is not difficult to check that for equilibria to exist generically, both loops defined by voltage sources, inductors and/or meminductors and cutsets composed of current sources, capacitors and/or memcapacitors must be precluded; this is a natural extension of the well-known exclusion of VL-loops and IC-cutsets for the existence of DC operating points (equilibria) in classical circuits [53], [54]. The exclusion of both configurations (which include in particular VL-loops and ICcutsets) guarantees that the geometric multiplicity of the aforementioned zero eigenvalue actually equals the total number of mem-devices, as stated below.

Theorem 3. Under the zero-crossing conditions (24), the absence of loops defined by voltage sources, inductors and/or meminductors and cutsets composed of current sources, capacitors and/or memcapacitors in a memcircuit with (positive) definite conductance $G$ implies that the geometric multiplicity of the zero eigenvalue at any given equilibrium of (17) equals the total number of $q$ and $\varphi$-memristors, memcapacitors and meminductors.

Proof. The absence of $q_{m}, q_{m l}, \varphi_{w}$ and $\varphi_{m c}$ from the equilibrium conditions implies that the geometric multiplicity of the zero eigenvalue is at least equal to the number of mem-devices. To check that it is actually equal, we need to show that the dimension of the kernel of the Jacobian matrix at equilibrium matches the number of mem-devices or, equivalently, that all circuit variables except $q_{m}, q_{m l}$, $\varphi_{w}, \varphi_{m c}$ must vanish for the corresponding vector to belong to this kernel. One can check that the kernel is defined by the conditions

$$
\begin{aligned}
& i_{c}=i_{m c}=i_{m}=i_{m l}=0 \\
& A_{l}^{T} e=A_{m l}^{T} e=A_{w}^{T} e=A_{m c}^{T} e=A_{u}^{T} e=A_{m}^{T} e=0 \\
& A_{u} i_{u}+A_{l} i_{l}+A_{g} G A_{g}^{T} e=0 .
\end{aligned}
$$

By premultiplying (25c) by $e^{T}$ one gets $e^{T} A_{g} G A_{g}^{T} e=0$, which in light of the definite nature of $G$ implies $A_{g}^{T} e=0$. Together with (25b) and the absence of IC-cutsets, this implies that $e=0$. In turn, this transforms (25c) into $A_{u} i_{u}+A_{l} i_{l}=0$, and the absence of VL-loops yields $i_{u}=i_{l}=0$. Finally, the vanishing of the remaining kernel variables $q_{c}=C A_{c}^{T} e, \varphi_{l}=L i_{l}, q_{m c}=C_{\mathrm{m}} A_{m c}^{T} e$ and $\varphi_{m l}=L_{\mathrm{m}} i_{m l}$ follows from that of $e, i_{l}, i_{m l}$ and the proof is complete.

\section{Nonlinear oscillators and Bifurcations}

We show in this Section how the fully nonlinear formalism introduced above allows for a general analysis of dynamical features in a broad family of nonlinear oscillators. The emphasis will be placed on the existence of a curve of equilibrium points, which will depend on the actual form of the mem-devices' fully nonlinear characteristics; we will also analyze certain associated bifurcation phenomena. Consider, specifically, the nonlinear R-L-C $\mathrm{C}_{m}$ circuit displayed in Fig. 5(a).

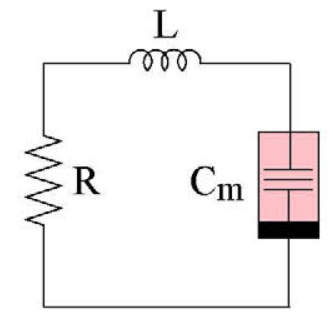

Fig. 5. (a) RLC mem-circuit

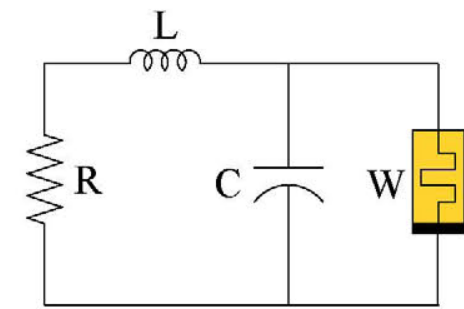

(b) Nonlinear oscillator of Itoh \& Chua.
In this RLC mem-circuit, the memory effect is given by the presence of a memcapacitor. Specifically, the circuit is composed of a linear resistor (with resistance $R$ ), a linear, strictly passive inductor (with inductance $L>0$ ) and a fully nonlinear memcapacitor governed by a general characteristic of the form $q=\omega(\varphi, v)$. All devices are assumed to be time-invariant. By differentiating the characteristic of the memcapacitor we may alternatively describe this device by means of the relation

$$
C(\varphi, v) v^{\prime}=-W(\varphi, v) v+i,
$$

where $C=\omega_{v}, W=\omega_{\varphi}$; note the coupling condition $C_{\varphi}(\varphi, v)=W_{v}(\varphi, v)$.

Depending on the form and the values of $\omega(\varphi, v)$ (or $C(\varphi, v)$ and $W(\varphi, v))$ and $R$, Fig. 5(a) accounts for several nonlinear circuits with distinct dynamical features. If $R>0, C>0$ and $W>0$, we get a passive R-L$\mathrm{C}_{\mathrm{m}}$ circuit. We will focus our attention mainly on two other cases: (i) with $R=0$ we arrive at a generalized tank circuit, used e.g. in quantum computation to couple flux qubits [35]; (ii) with $R<0$ we get self-sustained oscillations coming from the active nature of the resistor; in particular, if $\omega(\varphi, v)$ takes the form $C v+\xi(\varphi)$, the memcapacitor amounts to a parallel connection of a linear 
capacitor (with capacitance $C$ ) and a flux-controlled memristor of Chua type (with memductance $W(\varphi)=\xi^{\prime}(\varphi)$ ), yielding the nonlinear oscillator analyzed by Itoh and Chua in [19, Fig. 16] and displayed in Fig. 5(b) above.

Our aim is to provide a theoretical analysis of the circuit in Fig. 5(a) in general terms, accounting for the main dynamical properties displayed in these particular cases. The circuit equations can be written as

$$
\begin{aligned}
C(\varphi, v) v^{\prime} & =-W(\varphi, v) v+i \\
L i^{\prime} & =-v-R i \\
\varphi^{\prime} & =v .
\end{aligned}
$$

In broader generality, we will allow for a fully nonlinear characteristic $\zeta(\varphi, v)$ in (26a), directing the analysis to a general system of the form

$$
\begin{aligned}
C(\varphi, v) v^{\prime} & =-\zeta(\varphi, v)+i \\
L i^{\prime} & =-v-R i \\
\varphi^{\prime} & =v .
\end{aligned}
$$

Note that with $\zeta(\varphi, v)=W(\varphi, v) v$ we get (26).

Spectral analysis. The state-space dimension of system (27) is three, and therefore the eigenvalues at any equilibrium will be defined by a polynomial of the form

$$
\lambda^{3}+a_{2} \lambda^{2}+a_{1} \lambda+a_{0} .
$$

In the sequel we derive conditions for the vanishing of the different coefficients of this polynomial, which will determine the location of the eigenvalues and therefore will be responsible for different dynamic phenomena. Note that the goal is to perform the analysis in terms of the fully nonlinear form of the different devices involved.

Curve of equilibria: $a_{0}=0$. By considering the fully nonlinear characteristic $\zeta(\varphi, v)$ in (26a), we may have situations in which $a_{0}$ in (28) is not null and therefore no zero eigenvalue is displayed, meaning that the circuit displays isolated equilibria. This may happen if we disregard the zero-crossing conditions (24). Indeed, if the derivative $\zeta_{\varphi}$ does not vanish at a given equilibrium, the characteristic polynomial at equilibrium would read as

$$
\begin{aligned}
& \lambda^{3}+\left(C^{-1} \zeta_{v}+L^{-1} R\right) \lambda^{2}+ \\
& \quad+\left[L^{-1} C^{-1}\left(R \zeta_{v}+1\right)+C^{-1} \zeta_{\varphi}\right] \lambda+R L^{-1} C^{-1} \zeta_{\varphi} .
\end{aligned}
$$

The fact that the independent term $R L^{-1} C^{-1} \zeta_{\varphi}$ does not vanish implies that zero is not an eigenvalue or, equivalently, that the local dynamics has three non-null eigenvalues. Chaotic phenomena might therefore show up, defining a topic for future study. The reader is referred to [21], [24], [25], [26], [27] for somewhat related qualitative analyses of memristive circuits.

We will assume in the sequel that $\zeta(\varphi, v)=W(\varphi, v) v$, hence making the derivative $\zeta_{\varphi}$ vanish at equilibrium. Regardless of the values and the form of $C, W, L, R$, the circuit exhibits a curve of equilibria given by $v=i=0$ and parametrized by $\varphi$. As discussed in subsection III-D, these non-isolated equilibria define a salient feature of memristive circuit dynamics, and their local properties are discussed in what follows. The polynomial (29) now reads as

$$
\lambda\left[\lambda^{2}+\left(C^{-1} W+L^{-1} R\right) \lambda+L^{-1} C^{-1}(R W+1)\right],
$$

so that $a_{2}=C^{-1} W+L^{-1} R, a_{1}=L^{-1} C^{-1}(R W+1)$, $a_{0}=0$. Note that in this setting one eigenvalue always vanishes, consistently with the presence of an equilibrium curve. As the flux $\varphi$ varies along this curve, the memductance $W$ may undergo critical values yielding stability changes; in the cases considered below, we will address certain bifurcations stemming, specifically, from the vanishing of $a_{1}$ or $a_{2}$.

A transcritical bifurcation without parameters: $a_{1}=$ $0, a_{0}=0$. Assume that for certain parameter values, not only $a_{0}$ but also the coefficient $a_{1}$ does vanish, leading to a double zero eigenvalue. The additional degeneracy requirement $a_{1}=0$ can be expressed in graph-theoretic terms, by considering the reduced circuit used to locate the DC operating points, that is, the circuit obtained after short-circuiting inductors and open-circuiting capacitors. Omitting technical details, along the lines of Theorem 3 and by using a determinantal expansion of the node admittance matrix (cf. [55]) for such reduced circuit, the increase in the algebraic multiplicity of the zero eigenvalue may be shown to require the vanishing of the sum of admittance products in the spanning trees of the (reduced) circuit. In particular, for the circuit in Fig. 5(b) this reads $W+R^{-1}=0$, that is, $R W+1=0$. Note that, indeed, this is the condition for the coefficient $a_{1}=L^{-1} C^{-1}(R W+1)$ in (30) to vanish. As indicated in the setting of Case 2 below, this will be responsible for a transcritical bifurcation without parameters (cf. [56]).

Hopf bifurcation: $a_{2}=0, a_{0}=0$. Provided that $a_{2}=0, a_{0}=0$, the polynomial (30) displays a pair of purely imaginary eigenvalues besides a null one. In the classical Hopf bifurcation, the transition of a pair of conjugate eigenvalues through the imaginary axis along an equilibrium curve generically yields a change of stability in the equilibrium; additionally, either an asymptotically stable limit cycle shows up in the region of unstable equilibria, or an unstable periodic solution encircling stable equilibria is displayed. The Hopf bifurcation is called supercritical in the former case and subcritical in the latter. This type of phenomena will be displayed in our case if $W$ takes on a value for which $a_{2}=C^{-1} W+L^{-1} R$ vanishes, yielding a pair of purely imaginary eigenvalues; in particular, a supercritical Hopf bifurcation will arise in Case 1 below. This condition will also explain certain transitions in the nonlinear oscillator of Itoh and Chua analyzed in Case 2.

Case 1: a generalized tank circuit. Consider the case $R=0$ in Fig. 5(a). Short-circuiting the resistor yields a 
generalized tank circuit, with the eigenvalues along the equilibrium curve being given by

$$
\lambda_{1}=0, \lambda_{2,3}=\frac{-C^{-1} W \pm \sqrt{\left(C^{-1} W\right)^{2}-4(L C)^{-1}}}{2} .
$$

If $W$ vanishes at a given point of the equilibrium curve (say at $\varphi=0$ ), a pair of purely imaginary eigenvalues shows up and a stability change is expected to occur generically because of the transition of the eigenvalues through the imaginary axis. This is a particular case of the condition $a_{2}=C^{-1} W+L^{-1} R=0$ mentioned above.

Assume that $W(0,0)=0, W_{\varphi}(0,0)<0$, and let us examine what happens as $\varphi$ increases from negative to positive values. At $v=0$, this makes the memductance become negative, so that the memristor becomes locally active; the equilibrium at the origin turns from stable to unstable and a Hopf bifurcation is expected to occur.

Computer simulations show that a supercritical Hopf bifurcation is actually displayed, taking e.g.

$$
\omega(\varphi, v)=\frac{-\varphi^{2}}{2}+\frac{\varphi^{3} v}{3}+C_{0} v
$$

so that

$$
C=\frac{\varphi^{3}}{3}+C_{0}, W=-\varphi+v \varphi^{2} .
$$

System (26) admits, when $R=0$, a foliation by invariant surfaces of the form $L i+\varphi=\mu \in \mathbb{R}$. We may then recast (26) in terms of the parameter $\mu$; notice that $\mu=\varphi$ at equilibrium (where $i=0$ ). An asymptotically stable periodic solution encircling the origin shows up, indeed, for positive values of $\mu$; Fig. 6 displays this limit cycle, using the expressions depicted in (31) (with $C_{0}=1$ ), for the parameter values $\mu=0.001$ and $\mu=0.01$, respectively.

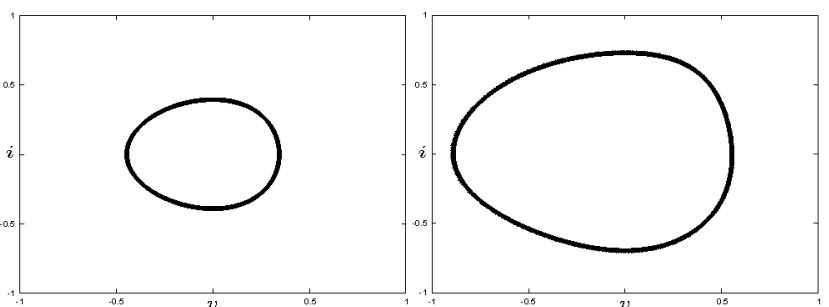

Fig. 6. Supercritical Hopf bifurcation:limit cycles: $\mu=0.001, \mu=0.01$.

Case 2: nonlinear oscillators with an active resistor. Let us now consider the case $R<0$ in Fig. 5(a). The resistor becomes active and this may also yield self-sustained oscillations, depending on the remaining parameter values.

Let us illustrate this by means of the nonlinear oscillator of Itoh and Chua displayed in Fig. 5(b). In [19], the authors consider a piecewise constant memductance $W$, and report a stability change from the value $W_{0}=0.02$ (yielding unstable equilibria) to $W_{1}=2$ (with stable equilibria); the remaining parameters are $C=L=1, R=-0.1$ [in the notation of [19] $a=W_{0}=0.02, b=W_{1}=2$,
$\left.\alpha=C^{-1}=1, \xi=L^{-1}=1, \beta=-R L^{-1}=0.1\right]$ We may explain this stability change by considering $W$ a parameter: with $C^{-1}=L^{-1}=1, R=-0.1$, the condition $a_{2}=C^{-1} W+L^{-1} R=0$ discussed above amounts to $W=0.1$; for $W<0.1$ (in particular for $W=W_{0}=0.02$ ) equilibria are unstable foci, which become stable (hence displaying stable periodic solutions) via a transition through the imaginary axis when $W$ undergoes the value 0.1 .

When $\left(C^{-1} W+L^{-1} R\right)^{2}=4 L^{-1} C^{-1}(R W+1)$ (this occurring at $W \approx 1.9$ in the case discussed by Itoh and Chua) the stable foci turn into stable nodes; this explains the stable node displayed when $W=W_{1}=2.0$ in [19].

As indicated above, the curve of equilibrium points arising in this problem is an instance of the manifolds of equilibria analyzed in subsection III-D. The fact that the qualitative changes occur along such a curve, without the presence of explicit parameters, place the results beyond the context of classical bifurcation theory. The analysis in this direction can be actually carried out further: indeed, the aforementioned condition $R W+1=0$ (this occurring for $W=10$ in the setting of [19] mentioned above, although this case was not analyzed by Itoh and Chua) would be responsible for a second zero eigenvalue. Generically, as $W$ crosses this critical value there would be a change of stability (in the context of [19], one real eigenvalue would reach the positive semiaxis, transforming the stable node into a saddle point). Although additional details are beyond the present paper, this is an instance of the transcritical bifurcation without parameters discussed in [56].

\section{FUtURE RESEARCH}

Several aspects remain open and define lines for future investigation on this topic. These include numerical issues, modeling aspects involving e.g. branch-oriented systems and hybrid analysis, or additional dynamical properties related to the nature of these circuits' operating points, their stability, bifurcations, as well as the eventual existence and characterization of chaotic effects. Higher order devices and mem-systems are also in the scope of future research.

In the long term, the notions and results discussed in this paper might be of interest as a modeling framework to accommodate present and future circuit elements, including nano-scale devices and even maybe molecular and biological devices [8]. It is expected that the future development of electronics (in a wide sense) will require broadening the theoretical foundations beyond the limits of classical circuit theory.

\section{ACKNOWLEDGEMENTS}

The author gratefully acknowledges several suggestions and bibliographic remarks from Professors M. Di Ventra (University of California, San Diego) and C. Tischendorf (University of Cologne, Germany). 


\section{REFERENCES}

[1] L. O. Chua, Memristor - The missing circuit element, IEEE Trans. Circuit Theory 18 (1971) 507-519.

[2] D. B. Strukov, G. S. Snider, D. R. Stewart and R. S. Williams, The missing memristor found, Nature $\mathbf{4 5 3}$ (2008) 80-83.

[3] L. O. Chua, Opening talk, Symposium on Memristors and Memristive Systems, Berkeley, Nov. 21, 2008.

[4] L. O. Chua, Introduction to memristors, IEEE Expert Now Educational Course, 2010

[5] M. Di Ventra, Y. V. Pershin and L. O. Chua, Circuit elements with memory: memristors, memcapacitors and meminductors, Proc. IEEE 97 (2009) 1717-1724.

[6] B. Mouttet, A memadmittance systems model for thin film memory materials, preprint, arXiv:1003.2842, 2010.

[7] Y. V. Pershin and M. Di Ventra, Memory effects in complex materials and nanoscale systems, $A d v$. Phys. 60 (2011) 145-227.

[8] L. O. Chua, Nonlinear circuit foundations for nanodevices, Part I: The four-element torus, Proc. IEEE 91 (2003) 1830-1859.

[9] K. E. Brenan, S. L. Campbell and L. R. Petzold, Numerical Solution of Initial-Value Problems in Differential-Algebraic Equations, SIAM, 1996.

[10] E. Hairer and G. Wanner, Solving Ordinary Differential Equations II: Stiff and Differential-Algebraic Problems, Springer-Verlag, 1996.

[11] P. Kunkel and V. Mehrmann, Differential-Algebraic Equations. Analysis and Numerical Solution, EMS, 2006

[12] R. Lamour, R. März and C. Tischendorf, Differential-Algebraic Equations: A Projector Based Analysis, Springer, 2012 (in press).

[13] P. J. Rabier and W. C. Rheinboldt, Theoretical and numerical analysis of differential-algebraic equations, Handbook of Numerical Analysis, Vol. VIII, 183-540, North-Holland (2002).

[14] R. Riaza, Differential-Algebraic Systems. Analytical Aspects and Circuit Applications, World Scientific, 2008.

[15] D. Estévez-Schwarz and C. Tischendorf, Structural analysis of electric circuits and consequences for MNA, Internat. J. Circuit Theory Appl. 28 (2000) 131-162.

[16] R. Riaza and C. Tischendorf, Semistate models of electrical circuits including memristors, Internat. J. Cir. Theory Appl. 39 (2011) 607627.

[17] C. Tischendorf, Topological index calculation of DAEs in circuit simulation, Surv. Math. Ind. 8 (1999) 187-199.

[18] C. Tischendorf, Coupled systems of differential algebraic and partial differential equations in circuit and device simulation. Modeling and numerical analysis, Habilitationsschrift, Humboldt-Univ. Berlin, 2003

[19] M. Itoh and L. O. Chua, Memristor oscillators, Internat. J. Bifurcation and Chaos 18 (2008) 3183-3206.

[20] R. Riaza, Dynamical properties of electrical circuits with fully nonlinear memristors, Nonlinear Analysis: Real World Applications 12 (2011) 3674-3686.

[21] B. Bao, Z. Ma, J. Xu, Z. Liu and Q. Xu, A simple memristor chaotic circuit with complex dynamics, Internat. J. Bifurcation and Chaos 21 (2011) 2629-2645.

[22] Z. Biolek, D. Biolek and V. Biolkova, Analytical solution of circuits employing voltage- and current-excited memristors, IEEE Trans. Circuits and Systems I, in press, 2012.

[23] M. Itoh and L. O. Chua, Memristor Hamiltonian circuits, Internat. J. Bifurcation and Chaos 21 (2011) 2395-2425.

[24] M. Messias, C. Nespoli and V. A. Botta, Hopf bifurcation from lines of equilibria without parameters in memristors oscillators, Internat. J. Bifurcation and Chaos 20 (2010) 437-450.

[25] B. Muthuswamy, Implementing memristor based chaotic circuits, Internat. J. Bifurcation and Chaos 20 (2010) 1335-1350.

[26] B. Muthuswamy and L. O. Chua, Simplest chaotic circuit, Internat. J. Bifurcation and Chaos 20 (2010) 1567-1580.

[27] B. Muthuswamy and P. P. Kokate, Memristor-based chaotic circuits, IETE Tech. Rev. 26 (2009) 417-429.

[28] Y. V. Pershin and M. Di Ventra, Practical approach to programmable analog circuits with memristors, IEEE Trans. Circuits and Systems I 57 (2010) 1857-1864.

[29] Y. V. Pershin and M. Di Ventra, Experimental demonstration of associative memory with memristive neural networks, Neural Networks 23 (2010) 881-886.
[30] R. Riaza, Nondegeneracy conditions for active memristive circuits, IEEE Trans. Circuits and Systems - II 57 (2010) 223-227.

[31] J. J. Yang, M. D. Pickett, X. Li, D. A. A. Ohlberg, D. R. Stewart and R. S. Williams, Memristive switching mechanism for metal/oxide/metal nanodevices, Nature Nanotechnology 3 (2008) 429-433.

[32] S. Adee, Memristor inside, IEEE Spectrum, Sept. 2010.

[33] F. Corinto, A. Ascoli and M. Gilli, Analysis of current-voltage characteristics for memristive elements in pattern recognition systems, Internat. J. Circuit Theory Appl. 40 (2012), in press.

[34] K. D. Cantley, A. Subramaniam, H. J. Stiegler, R. A. Chapman and E. M. Vogel, Neural learning circuits utilizing nano-crystalline silicon transistors and memristors, IEEE Trans. Neural Networks and Learning Systems 23 (2012) 565 - 573

[35] Y. V. Pershin and M. Di Ventra, Neuromorphic, digital and quantum computation with memory circuit elements, Proc. IEEE 100 (2012) 2071-2080.

[36] M. H. Devoret, A. Wallraff and J. M. Martinis, Superconducting qubits: A short review, 2004; http://arxiv.org/abs/condmat $/ 0411174$.

[37] D. Jeltsema and A. J. van der Schaft, Memristive port-Hamiltonian systems, Math. Comp. Model. Dyn. Sys. 16 (2010) 75-93.

[38] L. O. Chua, C. A. Desoer and E. S. Kuh, Linear and Nonlinear Circuits, McGraw-Hill, 1987.

[39] A. Brambilla, A. Premoli and G. Storti-Gajani, Recasting modified nodal analysis to improve reliability in numerical circuit simulation, IEEE Trans. Circuits and Systems I 52 (2005) 522-534.

[40] M. Günther and U. Feldmann, CAD-based electric-circuit modeling in industry. I: Mathematical structure and index of network equations, Surv. Math. Ind. 8 (1999) 97-129.

[41] T. Reis, Circuit synthesis of passive descriptor systems - a modified nodal approach, Internat. J. Circuit Theory Appl. 38 (2010) 44-68

[42] C. Sánchez-López, F. V. Fernández, E. Tlelo-Cuautle and S. X. Tan, Pathological element-based active device models and their application to symbolic analysis, IEEE Trans. Circuits and Systems I 58 (2011) 1382-1395.

[43] M. Takamatsu and S. Iwata, Index characterization of differentialalgebraic equations in hybrid analysis for circuit simulation, Internat. J. Circuit Theory Appl. 38 (2010) 419-440.

[44] R. März, Differential algebraic systems with properly stated leading term and MNA equations, in Modeling, Simulation, and Optimization of Integrated Circuits (Oberwolfach, 2001), Int. Ser. Numer Math. 146 (2003) 135-151.

[45] T. R. Bashkow, The A matrix, new network description, IRE Trans Circuit Theory 4 (1957) 117-119.

[46] P. R. Bryant, The order of complexity of electrical networks, Proceedings of the IEE, Part C $\mathbf{1 0 6}$ (1959) 174-188.

[47] P. R. Bryant, The explicit form of Bashkow's A matrix, IRE Trans. Circuit Theory 9 (1962) 303-306.

[48] Y.F. Lam, Formulation of normal form equations of nonlinear networks containing memristors and coupled elements, IEEE Trans Circuit Theory 19 (1972) 585-594.

[49] T. Martínez-Marín, State-space formulation for circuit analysis, IEEE Trans. on Education $\mathbf{5 3}$ (2010) 497-503.

[50] K. Yamamura and M. Tonokura, Formulating hybrid equations and state equations for nonlinear circuits using SPICE, Internat. $J$ Circuit Theory Appl., in press, 2012.

[51] R. A. Horn and Ch. R. Johnson, Matrix Analysis, Cambridge Univ Press, 1985.

[52] V. Venkatasubramanian, H. Schättler and J. Zaborszky, Local bifurcations and feasibility regions in differential-algebraic systems, IEEE Tr. Aut. Contr. 40 (1995) 1992-2013.

[53] B. C. Haggman and P. R. Bryant, Geometric properties of nonlinear networks containing capacitor-only cutsets and/or inductor-only loops. Part I: Conservation laws, Cir. Sys. Signal Process. 5 (1986) 279-319.

[54] T. Matsumoto, L. O. Chua and A. Makino, On the implications of capacitor-only cutsets and inductor-only loops in nonlinear networks, IEEE Trans. Circuits and Systems 26 (1979) 828-845.

[55] W. K. Chen, Net Theory and its Applications, World Scientific, 2003.

[56] B. Fiedler, S. Liebscher, and J. C. Alexander, Generic Hopf bifurcation from lines of equilibria without parameters: I. Theory, J. Differential Equations 167 (2000) 16-35. 